Full length article

\title{
Molecular composition of GAG-collagen I multilayers affects remodeling of terminal layers and osteogenic differentiation of adipose-derived stem cells
}

\author{
Mingyan Zhao ${ }^{\mathrm{a}, \mathrm{b}}$, George Altankov ${ }^{\mathrm{c}, \mathrm{d}}$, Urszula Grabiec ${ }^{\mathrm{e}}$, Mark Bennett ${ }^{\mathrm{f}}$, Manuel Salmeron-Sanchez ${ }^{\mathrm{f}}$, \\ Faramarz Dehghani ${ }^{\mathrm{e}}$, Thomas Groth ${ }^{\mathrm{b}, *}$ \\ a Stem Cell Research and Cellular Therapy Center, Affiliated Hospital of Guangdong Medical University, Renmin Dadao Road 57, Xiashan District, Zhanjiang 524001, China \\ ${ }^{\mathrm{b}}$ Biomedical Materials Group, Martin Luther University Halle-Wittenberg, Heinrich-Damerow-Strasse 4, Halle (Saale) 06120, Germany \\ ' Institut for Bioengineering of Catalonia, Baldiri Reixac, 10-12, Barcelona 08028, Spain \\ d ICREA (Institucio Catalana de Recerca i Estudis Avancats), Barcelona 08010, Spain \\ e Department of Anatomy and Cell Biology, Faculty of Medicine, Martin Luther University Halle-Wittenberg, Große Steinstraße 52, Halle (Saale) 06108, Germany \\ ${ }_{\mathrm{f}}^{\mathrm{f}}$ Division of Biomedical Engineering, School of Engineering, University of Glasgow, Glasgow, UK
}

\section{A R T I C L E I N F O}

\section{Article history:}

Received 29 February 2016

Received in revised form 3 May 2016

Accepted 13 May 2016

Available online $\mathrm{xxxx}$

\section{Keywords:}

Layer-by-layer technique

Glycosaminoglycans

Collagen reorganization

Mesenchymal stem cells

Osteogenic differentiation

\begin{abstract}
A B S T R A C T
The effect of molecular composition of multilayers, by pairing type I collagen ( $\mathrm{Col}$ I) with either hyaluronic acid (HA) or chondroitin sulfate (CS) was studied regarding the osteogenic differentiation of adhering human adipose-derived stem cells (hADSCs). Polyelectrolyte multilayer (PEM) formation was based primarily on ion pairing and on additional intrinsic cross-linking through imine bond formation with Col I replacing native by oxidized HA (oHA) or CS (oCS). Significant amounts of Col I fibrils were found on both native and oxidized CS-based PEMs, resulting in higher water contact angles and surface potential under physiological condition, while much less organized Col I was detected in either HA-based multilayers, which were more hydrophilic and negatively charged. An important finding was that hADSCs remodeled Col I at the terminal layers of PEMs by mechanical reorganization and pericellular proteolytic degradation, being more pronounced on CS-based PEMs. This was in accordance with the higher quantity of Col I deposition in this system, accompanied by more cell spreading, focal adhesions (FA) formation and significant $\alpha 2 \beta 1$ integrin recruitment compared to HA-based PEMs. Both CS-based PEMs caused also an increased fibronectin (FN) secretion and cell growth. Furthermore, significant calcium phosphate deposition, enhanced ALP, Col I and Runx2 expression were observed in hADSCs on CS-based PEMs, particularly on oCS-containing one. Overall, multilayer composition can be used to direct cell-matrix interactions, and hence stem cell fates showing for the first time that PEMs made of biogenic polyelectrolytes undergo significant remodeling of terminal protein layers, which seems to enable cells to form a more adequate extracellular matrix-like environment.
\end{abstract}

\section{Statement of Significance}

Natural polymer derived polyelectrolyte multilayers (PEMs) have been recently applied to adjust biomaterials to meet specific tissue demands. However, the effect of molecular composition of multilayers on both surface properties and cellular response, especially the fate of human adipose derived stem cells (hADSCs) upon osteogenic differentiation has not been studied extensively, yet. In addition, no studies exist that investigate a potential cell-dependent remodeling of PEMs made of extracellular matrix (ECM) components like collagens and glycosaminoglycans (GAGs). Furthermore, there is no knowledge whether the ability of cells to remodel PEM components may provide an added value regarding cell growth and differentiation. Finally, it has not been explored yet, how intrinsic cross-linking of ECM derived polyelectrolytes that improve the stability of PEMs will affect the differentiation potential of hADSCs. The current work aims to address these questions and found that the type of GAG has a strong effect on properties of multilayers and osteogenic differentiation of hADSCs. Additionally, we also show

\footnotetext{
* Corresponding author.

E-mail addresses: mingyan985927@163.com (M. Zhao), galtankov@ibecbarcelona.eu (G. Altankov), urszula.grabiec@medizin.uni-halle.de (U. Grabiec), m.bennett.

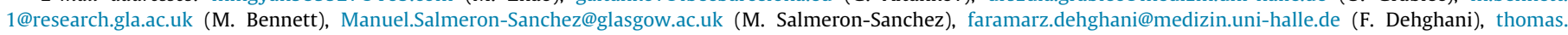
groth@pharmazie.uni-halle.de (T. Groth).
} 
for the first time that PEMs made of biogenic polyelectrolytes undergo significant remodeling of terminal layers as completely new finding, which allows cells to form an ECM-like environment supporting differentiation upon osteogenic lineage. The finding of this work may open new avenues of application of PEM systems made by layer by layer (LbL) technique in tissue engineering and regenerative medicine.

() 2016 Acta Materialia Inc. Published by Elsevier Ltd. All rights reserved.

\section{Introduction}

In native tissues, cells are tightly connected to the extracellular matrix (ECM), which regulates development, functioning and regeneration of tissues [1,2]. The ECM acts as physical support of cells and provides them with environmental signals, which determine cellular fates [3]. In particular, the molecular composition and specific structure of ECM regulate cellular processes, like adhesion, proliferation and differentiation [4-6]. Hence, the ECM plays a fundamental role during development and regeneration. Thereby, the cell-ECM interactions have a highly dynamic character, since cells continuously remodel the matrix changing this way the cellular microenvironment, which eventually affects cell behavior [7]. In this context, it was observed recently that cells remodel adsorbed collagens both by mechanical reorganization and proteolytic degradation [8-10]. It was also found that fibronectin (FN) possesses an important role for the mechanical reorganization of collagen [9-11]. Indeed, the relative amounts and organization of different ECM components vary from tissue to tissue, reflecting its peculiar functions. Hence, one aim of regenerative medicine is to design biomaterials that mimic the ECM composition of a specific tissue to guide cell differentiation and function into the desired lineage type.

Bone is a complex tissue with an intricate and ordered association of organic and inorganic components in a continuously renewing biological environment [12]. During the in vivo bone formation, mesenchymal stem cells (MSCs) derived osteoblasts synthesize and excrete type I collagen ( $\mathrm{Col} \mathrm{I}$ ) the main component of the organic matrix of bone, but also other specific bone matrix components, such as osteocalcin, proteoglycans, etc. The Col I molecular chains self-assemble in triple helical molecules, fibrils and three dimensional networks and become then mineralized with hydroxyapatite, which is embedded within the interstices and roughly aligned parallel to the long axis of the fibrils [12]. The spatial organization of Col I fibers in vivo is believed to play an important role in directing cell behavior and stem cell fate decisions [13]. Additionally, chondroitin sulfate (CS) from proteoglycans is another important macromolecule that is present at the early stage in bone and tooth formation, and found to be an effective promoter of hydroxyapatite nucleation and growth [14].

MSCs hold great promise for tissue engineering and regenerative medicine owing to their ability to differentiate into cell types of several mesenchymal tissues [15]. Particularly, human adiposederived stem cells (hADSCs) have gained an increasing attention since they are readily accessible in large amounts and possess the potential to form bone, cartilage, fat and other tissues $[16,17]$. Therefore, the successful application of hADSCs in bone tissue engineering is highly dependent on the capacity of culturing matrix-like environment to trigger a specific osteogenic response [18]. However, to provide such an environment at biomaterial's interface to cell and tissues is still a challenging task [19].

Indeed, chemical and physical surface modifications are frequently applied to address biomaterials to the specific tissue demands by binding ECM components like proteins or glycosaminoglycans (GAGs) to address specific cell receptors like integrins or growth factor receptors important for growth and differentiation of cells $[20,21]$. Among them, layer-by-layer (LbL) technique has emerged as a versatile method, which is based on alternating deposition of biogenic polyelectrolytes such as proteins, polypeptides and GAGs for formation of bioactive multilayer coatings [22,23]. Based on their opposite charge under acidic condition, Col I paired with either hyaluronic acid (HA) or CS represents an interesting system to create multilayers with a composition similar to connective tissue ECM [22]. Unfortunately, some of these biopolymer-based multilayers have been found relatively unstable under physiological conditions [24,25]. To enhance their stability, recently an intrinsic crosslinking between aldehydes of oxidized HA or CS and amino groups of Col I was introduced, resulting in enhanced stability and biocompatibility [22]. Here we continue studies with these multilayer systems looking at the ability of hADSCs to remodel the ECM-like multilayers during culture and the effect of multilayer composition and intrinsic cross-linking on differentiation of hADSCs under the effect of osteogenic growth media. Results are reported herein.

\section{Materials and methods}

\subsection{Preparation of polyelectrolyte solutions}

Poly (ethylene imine) (PEI, $\mathrm{M}_{\mathrm{w}} \sim 750 \mathrm{kDa}$, Sigma, Steinheim, Germany) was dissolved in a $0.15 \mathrm{M}$ sodium chloride (Roth, Karlsruhe, Germany) solution at a concentration of $5 \mathrm{mg} \mathrm{mL}^{-1}$. Native chondroitin sulfate $(\mathrm{nCS} \mathrm{Mw} \sim 25 \mathrm{kDa}$ ) was obtained from Sigma while native hyaluronic acid ( $\mathrm{nHA} \mathrm{Mw} \sim 1.3 \mathrm{MDa}$ ) was a kind gift from Innovent (Jena, Germany). Oxidized CS and HA (oCS and oHA) were prepared and characterized as described previously [22]. Native polyanion GAGs (nCS, nHA) and oxidized polyanion GAGs (oCS, oHA) were adjusted to a concentration of $0.5 \mathrm{mg} \mathrm{mL}^{-1}$. Type I collagen (Col I) from porcine skin (polycation, $\mathrm{M}_{\mathrm{w}} \sim 100 \mathrm{kDa}$, Sichuan Mingrang Bio-Tech, Sichuan, China) was used as polycation and dissolved in $0.2 \mathrm{M}$ acetic acid (Roth) at a concentration of $2 \mathrm{mg} \mathrm{mL}^{-1}$ at $4{ }^{\circ} \mathrm{C}$. After dissolution, the recovered solution was centrifuged at $9000 \mathrm{~g}$ for $10 \mathrm{~min}$ to remove any insoluble precipitates. A final concentration of $0.5 \mathrm{mg} \mathrm{mL}^{-1}$ was achieved by diluting the stock solution in $0.2 \mathrm{M}$ acetic acid supplement with sodium chloride (final concentration to $0.15 \mathrm{M}$ sodium chloride). Prior to use, the pH value of all polyelectrolyte solutions was adjusted to $\mathrm{pH} 4.0$ except for PEI ( $\mathrm{pH}$ adjusted to 7.4).

\subsection{Preparation of polyelectrolyte multilayers (PEMs)}

Polyelectrolyte multilayer (PEM) fabrication was performed on glass coverslips (Roth) of size $12 \times 12 \mathrm{~mm}$ and $15 \times 15 \mathrm{~mm}$, respectively. Prior to multilayer coating, glass coverslips were cleaned with $0.5 \mathrm{M}$ sodium hydroxide (Roth) dissolved in 96\% Ethanol (Roth) at room temperature for $2 \mathrm{~h}$ followed by extensive rinsing with ultrapure water and drying under nitrogen flow. Silicon wafers (Silicon materials, Kaufering, Germany) with a size of $(10 \times 10) \mathrm{mm}^{2}$ was treated with a solution of ammonium hydroxide (25\%, Roth), hydrogen peroxide (35\%, Roth) and ultrapure water $(1: 1: 5, \mathrm{v} / \mathrm{v} / \mathrm{v})$ at $75^{\circ} \mathrm{C}$ for $15 \mathrm{~min}$ followed by thoroughly washing with ultrapure water [26].

A first anchoring layer of PEI was formed on cleaned glass and silicon wafer (with its oxide layer on top) substrates to obtain a positive net surface charge, which was followed then by 
alternating adsorption of nGAGs (nCS, nHA) or oGAGs (oCS, oHA) as polyanions and $\mathrm{Col} I$ as polycation. The polyanions were adsorbed for $15 \mathrm{~min}$ at $\mathrm{pH} 4.0$, while Col I was adsorbed for $20 \mathrm{~min}$ at the same $\mathrm{pH}$ value. Each adsorption step was followed by three times rinsing with a solution of $0.15 \mathrm{M}$ sodium chloride $(\mathrm{pH} 4.0)$ for 5 min. Finally, Col I terminated multilayer systems with eight total layers (8th) on top of the PEI layer were obtained. The four different PEM systems were denoted as: nCS-Col I; nHA-Col I; oCS-Col I; and oHA-Col I.

\subsection{Characterization of polyelectrolyte multilayers (PEMs)}

\subsubsection{Measurement of type I collagen ( $\mathrm{Col}$ I) concentration in multilayers}

A protein quantification assay (BCA assay) was used to quantify the Col I content in the four PEM systems. In brief, PEMs coated glass coverslips were placed in 24-well plates (Greiner, Frickenhausen, Germany), followed by the reaction with $400 \mu \mathrm{L}$ working reagent of BCA Protein Assay Kit (Pierce, Rockford, USA) at $37^{\circ} \mathrm{C}$ for $5 \mathrm{~h}$. Thereafter, $225 \mu \mathrm{L}$ of supernatant from each well was carefully transferred to a new 96-well plate (Greiner), followed by measurement of absorbance at $562 \mathrm{~nm}$ with a plate reader (FLUOstar, BMG Lab Tech, Offenburg, Germany). The amount of Col I was determined from a calibration curve plotted from a series of Col I solutions with known concentrations.

\subsubsection{Detection of type I collagen (Col I) fibrils in multilayers}

AFM (Nano-R, Pacific Nanotechnology, Santa Clara, USA) was used to study the surface topography of Col I terminated multilayer surfaces. PEMs modified silicon wafers were incubated with PBS for $1 \mathrm{~h}$ and followed by drying with a flow of nitrogen. After that, all the samples were probed in a close-contact mode under ambient (air) laboratory conditions and scans of $(10 \times 10) \mu \mathrm{m}^{2}$ were recorded. The images were processed by using the software "Gwyddion 2.30".

\subsection{Cell culture}

Human adipose-derived stem cells (hADSCs) (Lonza, Walkwesville, USA) were grown in Dulbecco's modified Eagle's medium (DMEM/F12, Gibco, Alcobendas, Spain) supplied with $10 \%$ fetal bovine serum (FBS) and $1 \%$ penicillin-streptomycin solution (Gibco). Cells were detached from almost confluent flasks with $0.25 \%$ trypsin/0.02\% EDTA (Gibco). The remaining trypsin activity was stopped with $10 \%$ FBS containing DMEM/F12. The cells were resuspended in FBS-free medium after centrifugation at $800 \mathrm{~g}$ for 5 min. Finally, the cells were seeded on PEMs coated glass coverslips at a concentration of $2 \times 10^{4}$ cells $\mathrm{mL}^{-1}$.

\subsection{Short-term interaction of cells with multilayers}

\subsubsection{Quantification of hADSCS adhesion and spreading}

$500 \mu \mathrm{L}$ of cell suspension at a concentration of $2 \times 10^{4}$ cells $\mathrm{mL}^{-1}$ were seeded onto PEMs-coated glass slides for $1 \mathrm{~h}$ in serum free DMEM/F12. 10\% FBS was added at the end of the first hour considering the importance of serum proteins for the remodeling process, and incubated for another $3 \mathrm{~h}$ [27]. After incubation, samples were washed once with PBS and stained with crystal violet (Roth) $(0.5 \%(\mathrm{w} / \mathrm{v}))$ in methanol (Roth) at room temperature for 30 min. Finally, samples were carefully washed with ultrapure water and air dried. Images were taken in transmission mode with an Axiovert 100 (Carl Zeiss MicroImaging GmbH, Göttingen, Germany) equipped with a CCD camera (Sony, MC-3254, AVTHorn, Aalen, Germany). The number of adhering cells and the mean cell area were evaluated from five images per sample using image processing software "ImageJ, NIH, USA". Three samples were studied for each type of multilayers.

\subsubsection{Focal adhesion (FA) complex formation and actin organization}

hADSCs were seeded as described above. After $4 \mathrm{~h}$ incubation cells were fixed with $4 \%$ paraformaldehyde (Sigma) (10 min), permeabilized in $0.1 \%$ Triton X-100 in PBS (v/v) (Sigma) for another $10 \mathrm{~min}$ and blocked with PBS containing $1 \%(\mathrm{w} / \mathrm{v})$ bovine serum albumin (BSA, Sigma) for $30 \mathrm{~min}$ and washed finally with PBS. Focal adhesions (FA) were labelled using a monoclonal antivinculin antibody (1:400, Sigma) diluted in $1 \%(w / v)$ BSA in PBS at room temperature for 30 min followed by AlexaFluor 488 goat anti-mouse (1:400, InVitrogen, Engene, Oregon, USA) as a secondary antibody. The actin cytoskeleton was visualized at the same time by parallel incubation with AlexaFluor 555 Phalloidin (1:100, inVitrogen) for $30 \mathrm{~min}$, too. After washing with PBS and distilled water, samples were mounted with Mowiol (Merck, Darmstadt, Germany), examined and photographed with confocal laser scanning microscopy (CLSM 710, Carl Zeiss Micro-Imaging GmbH, Jena, Germany) using a $63 \times$ oil immersion objective. Images were processed with the ZEN2011 (Carl Zeiss).

2.5.2.1. Organization of cellular integrins. hADSCs were incubated and fixed as described above. To study the distribution of $\alpha_{2} \beta_{1}$ integrin, they were labelled by monoclonal anti-human integrin antibody of $\alpha_{2} \beta_{1}$ (1:100, Abcam, Cambridge, UK), followed by an incubation with goat-anti-mouse AlexFluor 488 as a secondary antibody. AlexaFluor 555 Phalloidin was mixed with the secondary antibody to stain the actin cytoskeleton. The incubation time for all antibodies and phalloidin was $30 \mathrm{~min}$. Samples were washed, mounted and viewed as described above. To quantify vinculin and $\alpha_{2} \beta_{1}$ integrin levels in cells, images were analyzed by Image J based on a reported method with some modification [28]. In brief, using ImageJ, an outline was drawn around each cell and area, mean and integrated intensity were measured. The background signal was taken from a region next to the cell of interest using a rectangular selection tool, and the area, mean and integrated density were calculated the same way. Then, the total corrected cellular fluorescence was calculated as integrated intensity minus the area of selected cells multiplied by mean fluorescence of background readings.

2.5.2.2. Remodeling of type I collagen (Col I) by hADSCs. hADSCs were seeded and incubated as described above for $4 \mathrm{~h}$ in the presence of $10 \%$ FBS. To study the fate of substratum adsorbed Col I, native Col I-containing PEM samples were processed for immunofluorescence using a monoclonal anti-Col I antibody (1:100, Sigma) followed by Cy2-conjugated goat anti mouse IgG (1:100, Dianova, Hamburg, Germany) as a secondary antibody. After washing with PBS and distilled water, samples were mounted and examined and photographed with CLSM using a $63 \times$ oil immersion objective. To assess the degree of rearrangement of Col I by hADSCs a method was adapted from previous work [29,30], with quantification being performed by ImageJ. Briefly, the images were first normalized to ensure the same total range of pixel values. The background intensity range of the Col I layer was taken from a defined region $(25 \times 25 \mu \mathrm{m})$ outside of the cell area for each condition. Across the samples the average of the minimum and maximum intensity values were used as thresholds to define the unaffected Col I layer. The cell area was then used as a mask over the Col I layer and the intensity values within the defined threshold were measured. The values obtained provided a\% area fraction of the selected area that was within the threshold values and was thus subtracted from $100 \%$ to indicate the amount of the Col I that has been rearranged.

To learn more about the fate of Col I layers at the internal part of PEMs, another approach was used to visualize Col I distribution via 
direct in-situ labelling with fluorescein isothiocyanate (FITC) dissolved in DMSO, a procedure which was described elsewhere [31]. Briefly, the FITC-labelled Col I was used for the initial 4 layers and then continuing layer formation with non-labelled Col I. To view simultaneously the cells, all samples, either native Col I or FITC-Col I -containing samples were further stained for actin with Alexa Fluor 555 Phalloidin as described above.

\subsubsection{Detection of fibronectin matrix and colocalization with type I collagen ( $\mathrm{Col} \mathrm{I}$ )}

To study the development of a FN matrix secreted and organized by hADSCs and FN colocalization with Col I, the cells were cultured for $4 \mathrm{~h}$ on native Col I or FITC-Col I -containing PEM surfaces. Native Col I was viewed as described above using Cy2conjugated goat anti mouse IgG as a secondary antibody, while secreted FN was viewed by a polyclonal anti-FN antibody (dilution 1:250, Sigma) followed by AlexaFluor 555-labelled anti-rabbit antibody (dilution 1:400, Invitrogen). All samples were finally mounted with Mowiol and examined with CLSM.

\subsubsection{Studies on cellular proteolytic activity}

Peri-cellular proteolytic activity was quantified in two levels: (i) by measuring the released fluorescent signal in the medium from FITC-labelled Col I samples (comparing with to without cells) following a protocol described previously [11] and (ii) measuring the signal from the extracted PEM- associated Col I (e.g. the protein that might be proteolytically altered but still remains adsorbed to the PEMs). For that purpose the Col I was labelled directly with FITC within the PEMs, as explained above, following the protocol described by McSwain et al. [31].

FITC-Col I containing PEMs prepared as above were seeded with hADSCs $\left(5 \times 10^{4}\right.$ cells per well, in 12 well-plates) and incubated overnight (first $1 \mathrm{~h}$ in serum-free medium to allow direct attachment to Col I on multilayers and then $10 \%$ serum was added). At the end of incubation supernatants were collected to measure the FITC-Col I release (first level) by samples that had been cultured either with or without cells using a fluorescent spectrometer (Fluoromax-4, HORIBA Scientific, Edison, USA) (488 nm excitation and $530 \mathrm{~nm}$ emission). Conversely, the PEM-associated FITC-Col I (second level) was measured after extraction of the protein with $0.2 \mathrm{M}$ sodium hydroxide (NaOH, $500 \mu \mathrm{L}$ per PEMs) for $2 \mathrm{~h}$ at $37^{\circ} \mathrm{C}$ from the samples comparing again samples with cells versus those without cells (e.g. control samples). Here we have to note that in preliminary studies we had observed that protein degradation by collagenase ( 20 unit $\mathrm{mL}^{-1}$ collagenase at $37^{\circ} \mathrm{C}$ for $2 \mathrm{~h}$ ) greatly increased the fluorescent yield of FITC-Col I, indicating that the labelled protein was quenched, presumably as a consequence of being labelled in excess of dye. We therefore speculated that eventual pericellular proteolysis, will cause de-quenching of FITC-Col I, e.g. will increase its fluorescence just as collagenase does, accepting the collagenase value as maximal protein degradation $(100 \%)$. Thus we obtained a scale varying between zero (sample without cells) and the maximal degradation (i.e. 100\% that is achieved by collagenase treatment), in which we could quantify the cellular proteolytic activity against Col I (samples with cells). More specifically we measured the fluorescent intensity of $\mathrm{NaOH}-$ extracts obtained from a given FITC labelled PEMs, cultured either with or without cells, and quantified the amount of its dequenching (increase of fluorescence) in the scale between zero (no cells) and $100 \%$ (collagenase activity). FITC-Col I samples were treated with collagenase (200 unit $\mathrm{mL}^{-1}$ from stock solution diluted with fresh medium at a ratio of $1: 10$ ) for $2 \mathrm{~h}$, which was accepted as positive control as explained above. All experiments were run in triplicates.
2.6. Study of hADSCs proliferation on type I collagen (Col I) terminated multilayers

One $\mathrm{mL}$ serum-containing hADSCs suspension $\left(1 \times 10^{4}\right.$ cells $\mathrm{mL}^{-1}$ ) was seeded on each PEM sample. The quantity of metabolic active cells after 1, 2 and 3 days was measured with a QBlue cell viability assay kit (BioChain, Newark, USA). Briefly, the medium was carefully aspirated and cells were washed once with sterile PBS. Then, $300 \mu \mathrm{L}$ of pre-warmed colorless DMEM supplemented with QBlue assay reagent (ratio of $10: 1$ ) were added to each well, followed by incubation for another $2 \mathrm{~h}$. Thereafter, $100 \mu \mathrm{L}$ supernatants were transferred to a black 96-well plate (Greiner) and fluorescence signals were measured at an excitation wavelength of $544 \mathrm{~nm}$ and emission wavelength of $590 \mathrm{~nm}$ with a plate reader.

\subsection{Induction of osteogenic differentiation}

Osteogenic differentiation was induced after a confluent monolayer was formed. The osteogenic differentiation media (OM) consisted of basal medium (BM, 1\% FBS and 1\% penicillinstreptomycin-containing DMEM) supplemented with osteogenic supplements namely, $10 \mathrm{nM}$ dexamethasone (Sigma), $50 \mathrm{mg} \mathrm{mL}^{-1}$ ascorbic acid (Sigma), $10 \mathrm{mM}$-glycerophosphate (Alfa Aesar, Ward Hill, USA) and $50 \mathrm{ng} \mathrm{mL}^{-1}$ BMP2 (Peprotech, Rocky Hill, USA). The medium was changed every 3 days. Each experiment was done in triplicate. The samples on which cells were cultured with BM have been used as reference.

\subsection{Detection of osteogenic differentiation of hADSCS}

\subsubsection{Activity of alkaline phosphatase (ALP)}

Alkaline phosphatase activity (ALP) was measured to evaluate the osteoblastic differentiation. Cell lysates obtained by treatment with $0.2 \%$ Triton $\mathrm{X}-100$ (Sigma) (in $10 \mathrm{mM}$ Tris- $\mathrm{HCl}(\mathrm{pH}=7.2)$ ) were incubated in substrate solution $(2 \mathrm{mM}$ p-nitrophenyl phosphate (pNPP, Sigma), in a substrate buffer (1 M diethanolamine at $\mathrm{pH} 9.8$ ) for $30 \mathrm{~min}$ in the dark at $37^{\circ} \mathrm{C}$, and measured the absorbance at $405 \mathrm{~nm}$ with a plate reader. The total protein content of the lysates was investigated by BCA assay. ALP activity was determined by normalization to the protein content of the lysates.

\subsubsection{Histochemical and immunofluorescence staining}

On day 21 post-differentiation, calcium phosphate deposition was investigated by Alizarin Red S staining. Staining with Alizarin Red S (2\%, pH 4.2, Roth) was performed for $45 \mathrm{~min}$ in the dark. Briefly, the samples were washed once with PBS and fixed with $4 \%$ paraformaldehyde for $10 \mathrm{~min}$. After twice washing with distilled water, Alizarin Red solution was added into each well, left working for $45 \mathrm{~min}$ in the dark at room temperature. Finally, the excess dye was removed by washing with distilled water. Images were taken in transmission mode with an Axiovert 100 equipped with a CCD camera.

To further investigate the development of a Col I matrix secreted by hADSCs, the samples were stained with a monoclonal anti-Col I antibody followed by Cy2-conjugated goat anti mouse IgG as a secondary antibody. To view the cells, samples were further stained for nuclear with To-Pro-3 (1:500, Invitrogen), mounted with Mowiol and examined with CLSM.

\subsubsection{Gene expression of osteogenic markers by real-time RT-PCR}

The expression levels of major osteogenic markers including Runx2 and Col I (Col IA) were used to evaluate whether the hADSCs differentiated into cells of the osteogenic lineage. On day 14 postdifferentiation, cells were detached from test samples using $0.25 \%$ trypsin/0.02\% EDTA. Total RNA was isolated from cells using Trifast 
Table 1

Primers used for qRT-PCR.

\begin{tabular}{|c|c|c|c|}
\hline & Forward primer & Reverse primer & Size \\
\hline$\beta$-actin & ACTCCTACGTGGGCGACGAGG & CAGGTCCAGACGCAGGATGGC & 389 bp \\
\hline Col IA & GCCAAGACGAAGACATCCCA & CACCATCATTTCCACGAGCA & $891 \mathrm{bp}$ \\
\hline Runx 2 & СТСАСТАССАСАССТАССТG & TCAATATGGTCGCCAAACAGATTC & $320 \mathrm{bp}$ \\
\hline
\end{tabular}

(Peq Lab, Erlangen, Germany) following standard protocols [32]. After DNAse treatment (DNase-Kit DNA-free, Life Technologies, Darmstadt, Germany) the extracted RNA was re-suspended in nuclease-free water (Promega, Madison, WI, USA) and the concentration was measured by spectrophotometry. cDNA was synthesized from $1 \mu \mathrm{g}$ of total RNA by reverse transcription using transcription system (Promega, Mannheim, Germany). PCR samples contained $10 \mu \mathrm{L}$ of Mastermix (Taq Polymerase $50 \mathrm{U} \mathrm{mL}^{-1}$, $\mathrm{MgCl}_{2}$, Nucleotids, Promega), $0.5 \mu \mathrm{L}$ of each primer, $0.25 \mu \mathrm{L}$ Eva Green (Biotium, Hayward, CA, USA), $4.75 \mu \mathrm{L}$ DNase free water and $4 \mu \mathrm{L}$ cDNA template. Reactions were performed on a Thermocycler (Rotor-GeneTM RG 6000, Corbett Research, Pty Ltd., Sydney, Australia). The expression of $m R N A$ in the cells was assessed by qRT-PCR by the use of specific primers (Table 1 ) and $\beta$-actin was used as the endogenous control. The data was analyzed by $2^{\wedge}$ ddct method following Livak et al. [33].

\subsection{Data analysis and statistics}

All quantitative data were expressed as the mean \pm standard error unless otherwise specified. Statistical analysis was performed using Origin software (Originlab Corporation, Northampton, USA) with ANOVA test (One way) followed by post Tukey testing. The number of samples is indicated in the respective figure and table captions. Statistical significance was considered for $\mathrm{p}<0.05$ and is indicated by asterisks.

\section{Results and discussion}

\subsection{Physicochemical characterization of polyelectrolyte multilayers (PEMs)}

While a more comprehensive characterization of the multilayer formation process and also the presence of intrinsic cross-linking were shown in a previous paper [22], here we show in Table 2 only an summary of surface properties and layer thickness taken from this work, because these are also determinants for the interaction with MSCs [34].
The measurement of WCA with the sessile drop method showed that multilayers with CS were significantly less hydrophilic than those with HA as polyanion. A potential reason for this observation could be a higher quantity of Col I in CS-based PEMs in comparison to HA-based systems, which was confirmed by the BCA assay. Table 3 shows that the amount of Col I in CS-based systems was almost twice as high as in HA-based PEMs probably due to the higher charge density of CS. The use of oxidized GAG led to slightly lower WCA, which was only significant when comparing PEMs made of either nHA/Col I or oHA/Col I. However, as found in the BCA assay, there was no significant difference in the amount of Col I either using the native or oxidized GAGs. Therefore, one reason for the higher hydrophilicity of the cross-linked systems might be a stabilization of GAG binding to surface regions of PEMs, which increases the wettability due to the more hydrophilic nature of GAGs in comparison to Col I. Zeta potentials, shown here only at pH 7.4 further support that more Col I polycation was present in CS-based systems due to lesser negative potentials compared to HA-containing PEMs. The finding that zeta potentials were less negative for oGAG based systems compared to PEM made of native GAG seems to be contradictory to WCA measurements at a first view. However, as described in more detail in our previous paper and also shown by others, zeta potentials of PEMs are not only a representation of the outermost polyelectrolyte layer, but also of intrinsic composition of layers closer to the surface [35,36]. Therefore, it may also be assumed from these measurements that the lesser negative zeta potentials of CS-based PEMs indicate a more dominant role of the polycation Col I in this system. Ellipsometry measurements showed also that intrinsic cross-linking caused a higher layer thickness in both oCS- and oHA-based PEMs compared to PEMs made of native GAGs, which indicates that the covalent cross-linking leads to more binding of polyelectrolytes compared to ion pairing alone. Overall, the physical characterization of multilayer surface properties corresponds well to the different compositions of both systems and shows also that the intrinsic crosslinking by imine bond formation has only a small effect. However, as it will be lined out further in this manuscript, effects on cell adhesion, growth and differentiation are most probably centering

Table 2

Surface properties and thickness of type I collagen (Col I) terminated polyelectrolyte multilayer (PEM) systems.

\begin{tabular}{|c|c|c|c|}
\hline Multilayer systems & Water contact angle ${ }^{\mathrm{a}}\left({ }^{\circ}\right)$ & Zeta potential $^{\mathrm{b}}(\mathrm{mV})$ & Thickness $^{\mathrm{c}}(\mathrm{nm})$ \\
\hline nHA-Col I & $\sim 34.3^{\circ} \pm 1.7^{\circ}$ & $-57.5 \pm 0.3$ & $11.9 \pm 1.1$ \\
\hline oHA-Col I & $\sim 26.6^{\circ} \pm 1.6^{\circ}$ & $-42.3 \pm 1.9$ & $25.6 \pm 1.5$ \\
\hline nCS-Col I & $\sim 45.2^{\circ} \pm 1.4^{\circ}$ & $-44.0 \pm 0.5$ & $13.5 \pm 0.1$ \\
\hline oCS-Col I & $\sim 43.4^{\circ} \pm 3.4^{\circ} \mathrm{J}$ & $-37.5 \pm 1.1$ & $17.6 \pm 0.6$ \\
\hline
\end{tabular}

*Four bilayers of native hyaluronic acid (nHA), oxidized hyaluronic acid (oHA), native chondroitin sulfate (nCS) and oxidized chondroitin sulfate (oCS) were prepared with type I collagen (Col I) as polycation. ${ }^{\text {a Static }}$ water contact angles (WCA) for Col I terminated polyelectrolyte multilayers (PEMs) (8th layer). ( $n=16$, $* \mathrm{p}<0.05$ ). ${ }^{\mathrm{b}}$ Zeta potential of outermost Col I layer for multilayer systems measured at pH $7.4 \mathrm{in} 1 \mathrm{mM} \mathrm{KCL}$. 'Layer thickness was determined by ellipsometry in PBS buffer, pH 7.4. Data were extracted from results of our previous work [22]. 
Table 3

Type I collagen (Col I) content of polyelectrolyte multilayers (PEMs)*.

$\left.\left.\begin{array}{lc}\hline \text { Name of samples } & \text { Type I Collagen }\left(\mu \mathrm{g} \mathrm{cm}^{-2}\right) \\ \hline \text { nHA-Col I } & 10.2 \pm 1.3 \\ \text { oHA-Col I } & 9.9 \pm 2.2\end{array}\right]\right\} *$

*Four bilayers of native hyaluronic acid ( $\mathrm{nHA}$ ), oxidized hyaluronic acid (oHA), native chondroitin sulfate $(\mathrm{nCS})$ and oxidized chondroitin sulfate (oCS) were prepared with type I collagen (Col I) as polycation. ( $n=4$, $\left.{ }^{*} \mathrm{p}<0.05\right)$
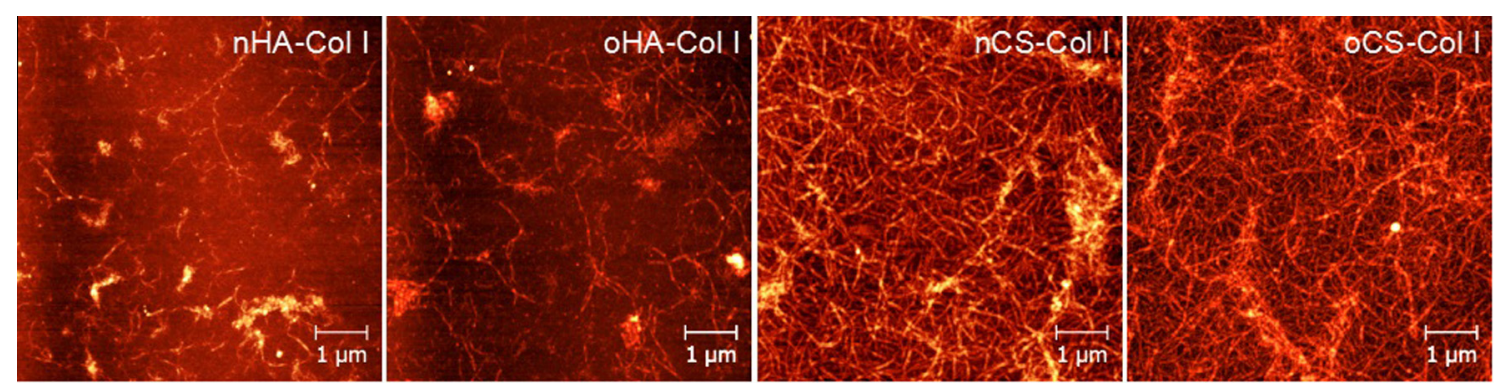

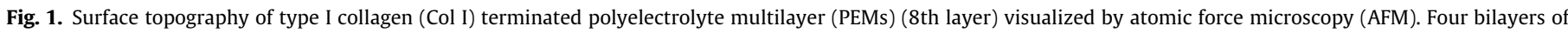

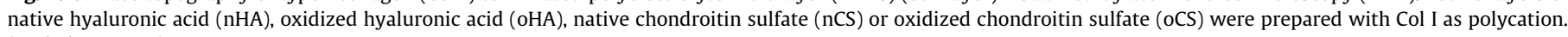
(Scale bar: $1 \mu \mathrm{m}$ ).

on the different content and organization of Col I in terminal layers since differences in wettability and surface potential were relatively small.

To learn more about the ability of GAG to support fibrillization of Col I in PEMs, multilayers were investigated with AFM (see Fig. 1). Col I aggregates were present in all multilayer systems, although a prominent fibrous structure was found mostly on CSbased PEMs (both nCS and oCS) with a tendency to form a network-like structure. Conversely, rather sparse and short fibrils were observed in both nHA and oHA-containing PEMs, where Col I seemed to form rather discrete aggregates than fibers. Col I fibrillogenesis is a sophisticated process highly affected by environmental factors, such as pH, electrolyte type and collagen concentration, but also on the presence of GAG like CS and other naturally occurring polyanions $[37,38]$. In general, at $\mathrm{pH}$ value $<5.5$ Col I forms more globular structures, while at $\mathrm{pH}$ value $>5.5$ normally fibrillogenesis is supported [39]. The presence of GAG however, particularly CS, seems to play an important role in regulating Col I fibril formation as it happened even at $\mathrm{pH} 4$. Also, the concentration of Col I affects strongly fibril formation as found previously [40,41]. Presumably CS binds to collagen molecules and facilitates the organization of mature fibrils by increasing collagen concentration as it was found in the presence of 4,6-disulfated disaccharides structures, previously [38]. In line with this, here a pronounced fibril formation in both nCS and oCS-based PEMs was observed, when more Col I was adsorbed, which showed also a specific networklike organization as in connective tissue.

\subsection{Short-term culture of hADSCs on multilayers}

hADSCs were seeded on the samples for $1 \mathrm{~h}$ in serum-free medium to allow a direct contact of cells with Col I on the multilayer surfaces avoiding the interference with other proteins. Thereafter,
$10 \%$ serum was added and samples were cultured for 3 more hours. The quantitative data of cell adhesion and spreading are shown in Fig. 2. No significant differences in cell adhesion were found, although cell number was slightly higher on CS-based PEMs independent on oxidization (see Fig. 2A), which could be related to the higher amount of fibrillar Col I and the lower wettability of these multilayers. By contrast, significantly higher extent of cell spreading was observed on CS-based (both nCS-Col I and oCS-Col I) multilayers compared to nHA-containing PEMs ( $p<0.05$ ) as shown in Fig. 2B. It was also observed that cells spread significantly more on oCS-containing PEMs compared to oHA-based one, while no significant difference was found between nCS-Col I and oHA-Col I.

To learn more about the organization of hADSCs adhesive machinery, we followed the formation of focal adhesions (FA), visualized by staining of vinculin and the development of actin cytoskeleton (viewed by fluorescence-labelled phalloidin) after $4 \mathrm{~h}$ of incubation (see Fig. 2C). Though a pronounced cell spreading was found on all samples, a remarkable difference was found in the overall appearance of FA plaques. Much less extended and rather dot-like FA plaques were found on HA-based PEMs, while larger and elongated FA plaques where longitudinal actin stress fibers often inserted (resulting in the yellow color of the merged images) were detected on CS-based PEMs, which is again well in line with the presence of more fibrillar Col I. In particular, the quantitative analysis of vinculin-positive focal adhesions showed higher quantities in cells plated on CS-based PEMs compared to HA-containing ones. In addition, the intrinsic cross-linking in multilayer systems made of oCS/Col I compared to nCS/Col I (as shown in Fig. 2D). Vinculin as part of FA complexes is directly involved in actin cytoskeleton organization, which is critical for the development of cell spreading. In addition the formation of FA complexes is also involved in signal transduction via FA and other kinases [42]. 
A

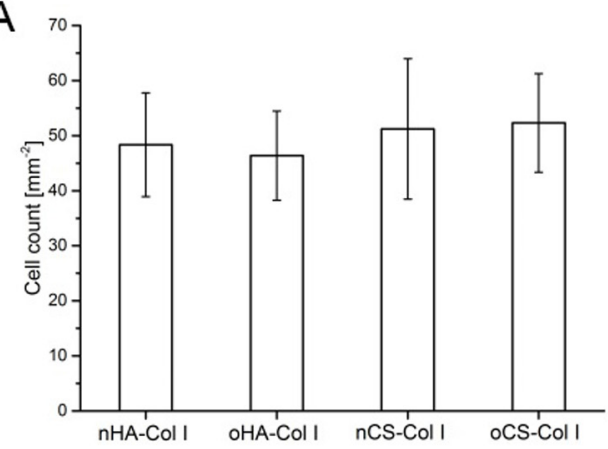

B

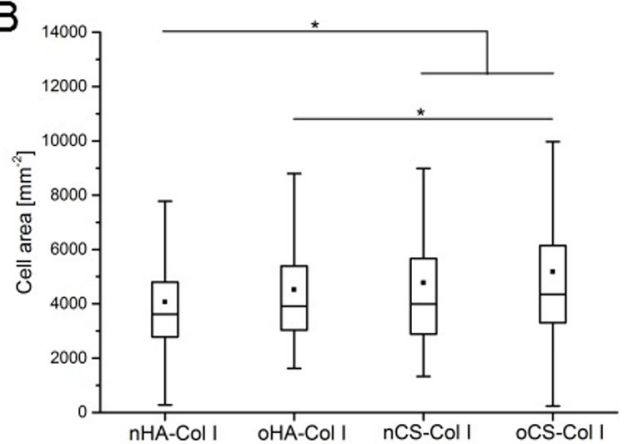

C

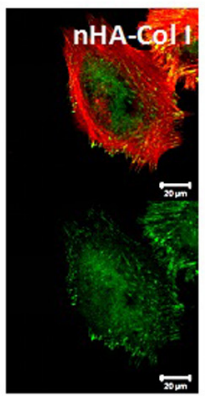

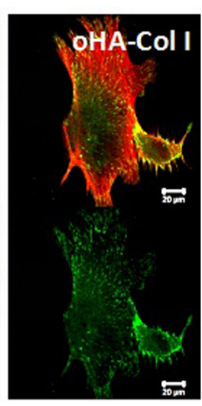
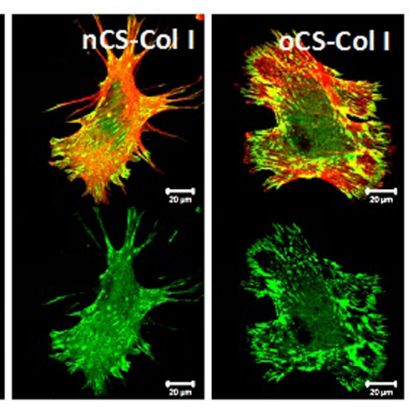

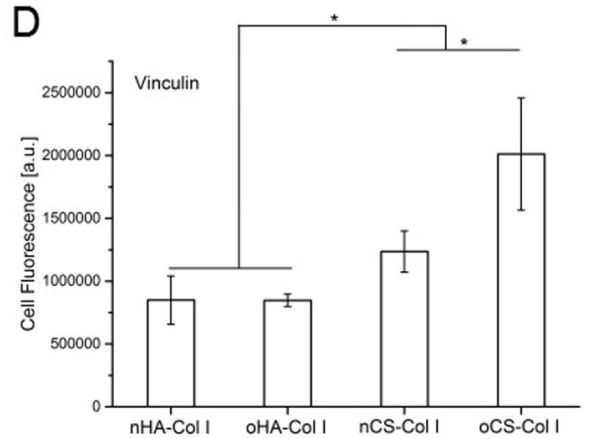

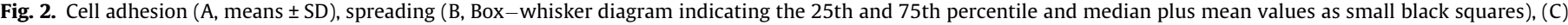

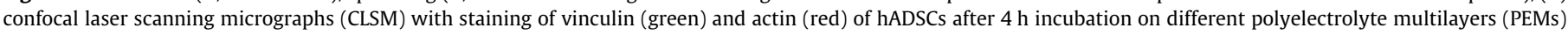

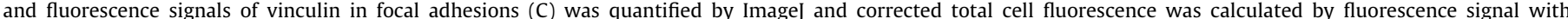

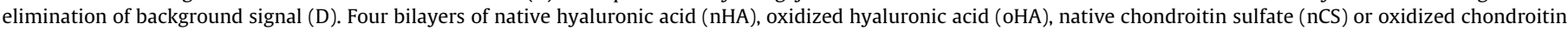

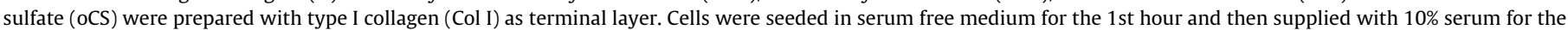
next $3 \mathrm{~h}$ of incubation. [Scale bar: $20 \mu \mathrm{m}$ ].

Since integrins are the major components of FA plaques connecting ECM proteins with the cell cytoskeleton and signaling complexes, we studied the expression, organization and quantification of $\alpha_{2} \beta_{1}$ that is regarded as main collagen receptors $[43,44]$. Indeed, as shown in Fig. 3A and B $\alpha_{2} \beta_{1}$ was more expressed and organized in hADSCs adhering on CS-based PEM surfaces (either nCS-Col I or oCS-Col I), forming clusters resembling small FA plaques. Conversely, on HA-based PEMs the cells showed a weaker integrin expression and a rather diffuse distribution with a tendency for accumulation of these integrins at the cell edges (see Fig. $3 \mathrm{~A}$ and $\mathrm{B}$ as well).

The generally stronger adhesion, spreading and integrin organization of cells on CS-containing PEMs was obviously related to the composition of these PEM systems. First, more Col I was present in the CS-based multilayers (proven by BCA assay), which formed a dense fibrillar network resembling the natural Col I environment. The presence of Col I fibrils is known to enhance the adhesion of a variety of cells via an $\alpha_{2} \beta_{1}$ integrin-mediated mechanism [45], which explains also the apparently lower organization of this integrin on HA-based PEMs that contained less and poorly fibrillized Col I. The amount of GAG obviously dominates in the HA-based systems as shown also in our previous work leading formation of smaller of Col I fibrils [22], which seemed to be poorly recognized by $\alpha_{2} \beta_{1}$ integrins. On the other hand, the increased hydrophilicity of HA-based PEMs may further hinder cell spreading due to the increased water content of the layers resulting from the ability of HA to bind large amounts of water [46]. It is also interesting to note that both spreading and organization of cell adhesion complexes was more pronounced on oCS-based PEMs compared to nCS-Col I PEMs. Since intrinsic cross-linking by imine bond formation leads to additional changes of multilayer properties like increased stiffness [22], effects on adhesion and spreading of cells can be expected [47].

\subsection{Remodeling of type I collagen (Col I)}

Stability of PEMs made of biogenic polyelectrolytes is discussed critically because of a possible degradation of biomolecules in the relatively "aggressive" biologic environment, which is rich in protein degrading enzymes like matrix metalloproteases released by cells [48]. On the other hand remodeling of adsorbed protein on biomaterials seems often to improve the biological performance of adhering cells $[11,28]$, which can be exploited as biomimetic approach for creating conditions closer to the natural tissue [49].

\subsubsection{Reorganization of type I collagen (Col I) by mechanical remodeling}

Fig. 4A presents the overall morphology of hADSCs at low magnification after $4 \mathrm{~h}$ of incubation viewed by actin staining on the background of adsorbed Col I visualized by monoclonal antibodies. The micrographs shows that the cells not only attach to the terminal layer of Col I on PEMs, but also tend to remove the protein from the substratum (dark zones) and arrange it into fibril-like pattern, particularly evident on CS-based PEMs. The reorganization is better visible at higher magnification shown on Fig. 4B where only Col I is shown. It should be underlined that this reorganization is not compromising stability of PEMs since the remodeling process affected only the outermost layers (Supporting information S1), suggesting that cells were not able to reach Col I in subzones of both native and oxidized GAG-based PEMs. In notable contrast, much less reorganization of Col I was found in HA-containing PEMs, especially on oHA-based PEMs, which is also related to the lower content of Col I in these PEMs.

These studies demonstrate for the first time that hADSCs are not only able to recognize substratum-associated Col I in PEMs utilizing their integrin receptors, but also to reorganize this protein in terminal layers of PEMs. It should be also noticed that a distinctly 

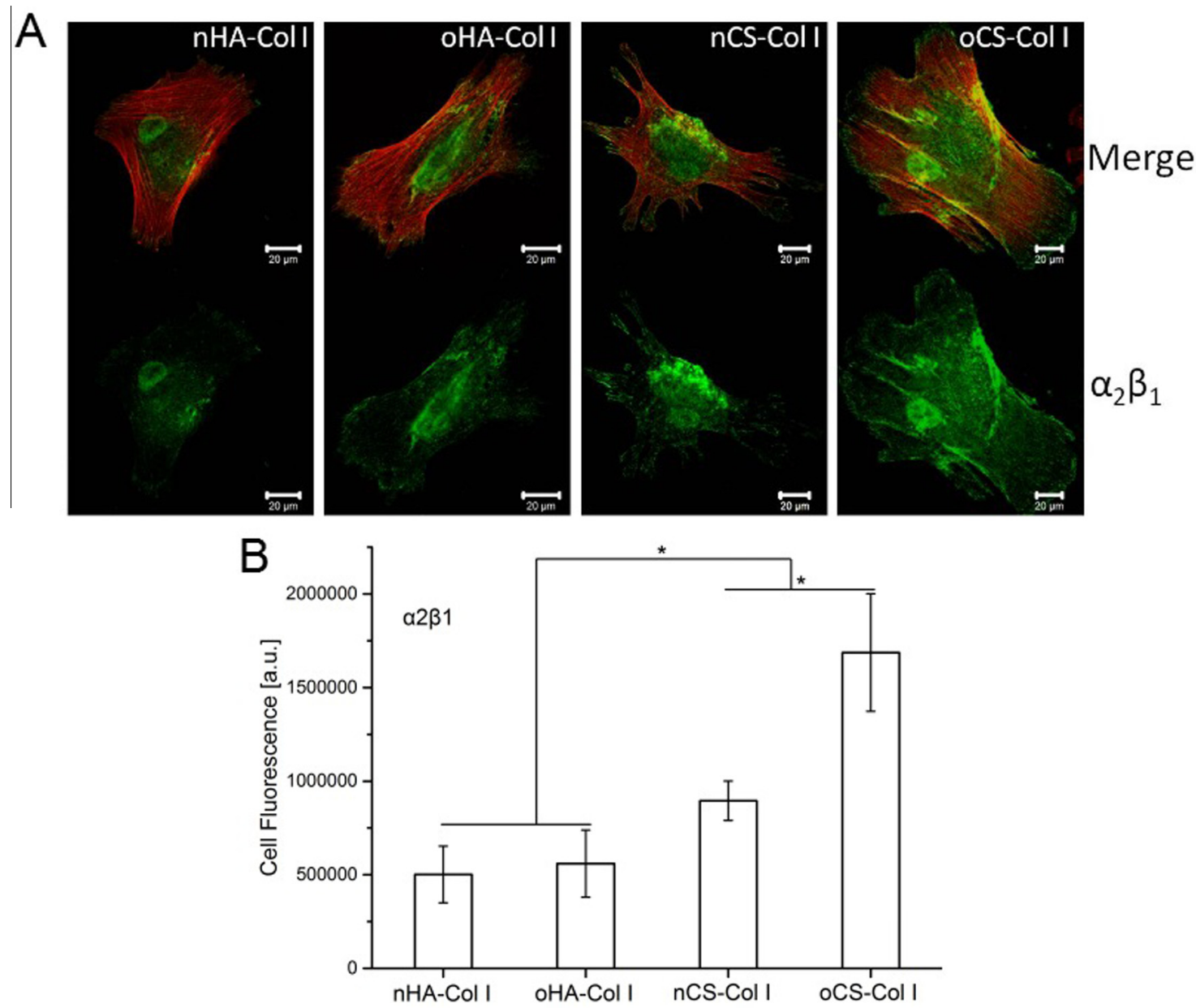

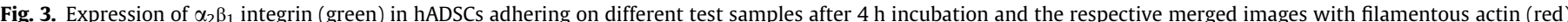

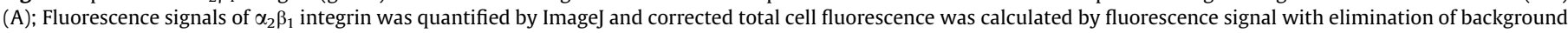

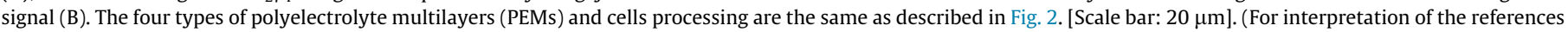
to colour in this figure legend, the reader is referred to the web version of this article.)

different extent of Col I rearrangement was found among the four types of PEMs (Fig. 4C). The apparently visible fibril-like pattern particularly on CS-containing PEMs clearly revealed the ability of hADSCs to remodel Col I involving integrins. Conversely, the less apparent reorganization of Col I on HA-based PEMs presumably is caused by the lowered amount of protein on this surface resulting in less binding sites for integrins leading to a weaker cellular interaction manifested by less FA formation and diminished accumulation of $\alpha_{2} \beta_{1}$ integrin. An interesting observation was the less pronounced reorganization of Col I on oGAG-based PEMs, which is presumably owing to the increased stability of the Col I layer by intrinsic cross-linking [22], as an additional evidence for the increased overall stability of this type of PEMs, detected also previously with other methods [22].

FN molecules possess two binding sites for collagens [50] and have been demonstrated to drive the organization of collagen type I and III into fibrils [51,52]. Fibril-like reorganization in the presence of cells was found also for the non-fibrillar collagen type IV $[9,11]$. Interestingly, co-staining of FN with Col I in this study showed significant FN secretion on CS-based PEMs in comparison to HA-containing multilayers (see Fig. 5). This indicates an increased activity of hADSCs on CS-based PEMs by means of de novo synthesis of FN. Furthermore, the clearly expressed colocalization of FN with Col I (resulting in orange on merges seen in the upper panel in Fig. 5) indicates a close relationship between
FN fibrillogenesis and mechanical remodeling of Col I. It has to be noted however that a large part of Col I appears still green, which points to the possibility of an additional, most probably also integrin-dependent mechanisms contributing to the reorganization process. This is in agreement with other studies showing that integrin signaling regulates ECM organization and remodeling and thus contributes to the control of cell behavior in tissues [53]. Also the enhanced $\alpha_{2} \beta_{1}$ integrin clustering on CS-containing PEMs, where cells arranged more Col I fibrils is well in line with this (see Fig. 3). On the other hand, cross-linking of proteins has been shown to inhibit reorganization processes [54], which explains the reduced Col I reorganization on crosslinked PEMs using oxidized GAG shown in the present study.

\subsubsection{Proteolytic remodeling of FITC labelled type I collagen (FITC-Col I)}

Apart from the mechanical tethering of Col I by the cells, another potential mechanism of Col I remodeling is the pericellular proteolysis by secretion of metallomatrix proteinases (MMPs) [8]. Here the dark zone around cells in Figs. 4 and 5 represents the places from where the protein was removed to be arranged mechanically in a fibril-like pattern, but possibly also due to a proteolytic degradation of adsorbed Col I [9,11]. To study the contribution of proteolytic degradation and to learn also about stability of these PEMs, FITC-Col I was used to quantify the cellular proteolytic 

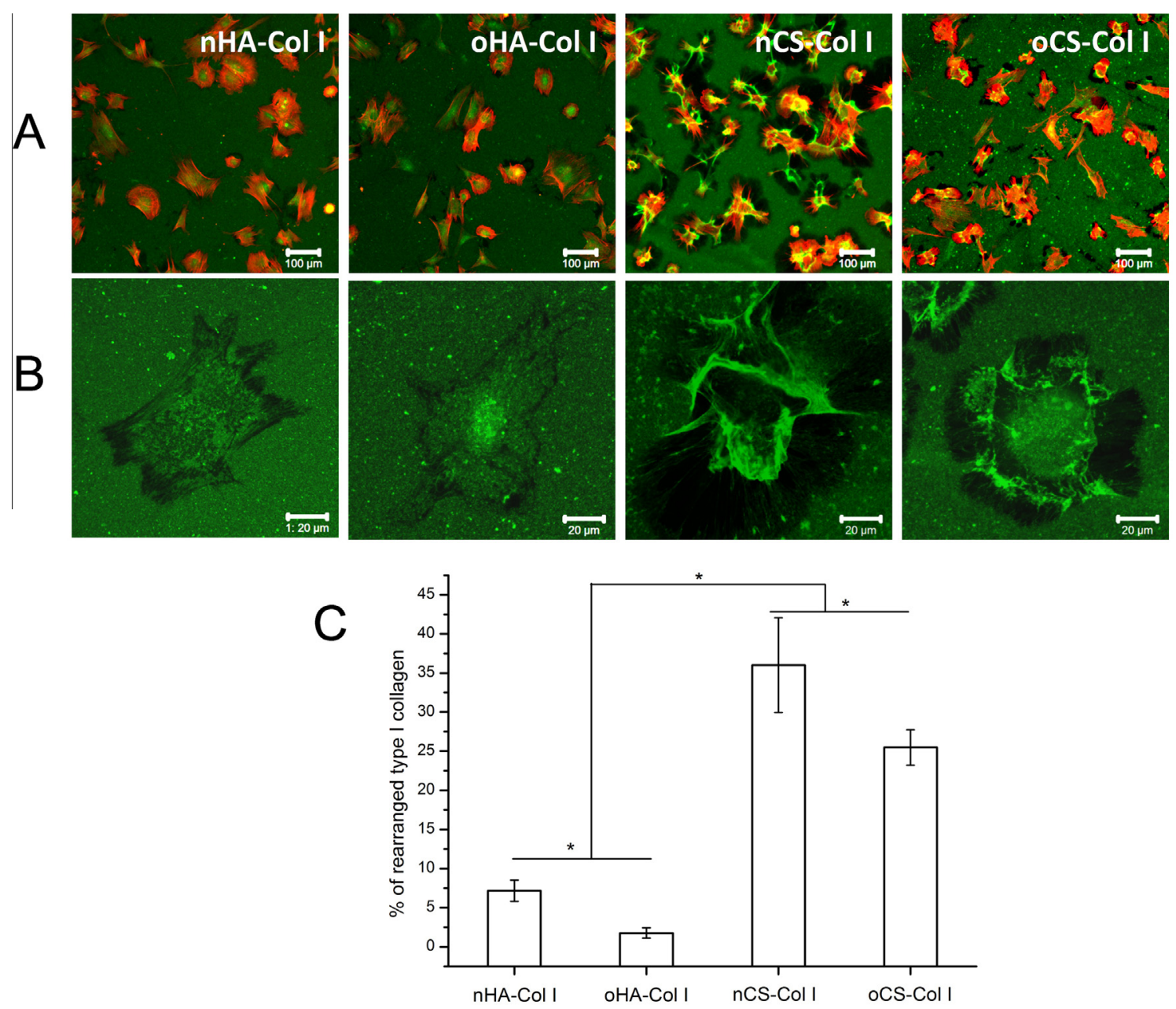

Fig. 4. Remodeling of type I collagen ( $\mathrm{Col} \mathrm{I}$ ) (green) on the different polyelectrolyte multilayers (PEMs) by adhering hADSCs (stained for actin in red) (A, B) and the quantification of Col I rearrangement (C). Col I is visualized by anti-Col I antibody, the four types of polyelectrolyte multilayers (PEMs) and cell processing are the same as described in Fig. 2. [Scale bar: $20 \mu \mathrm{m}$ ]. (For interpretation of the references to colour in this figure legend, the reader is referred to the web version of this article.)
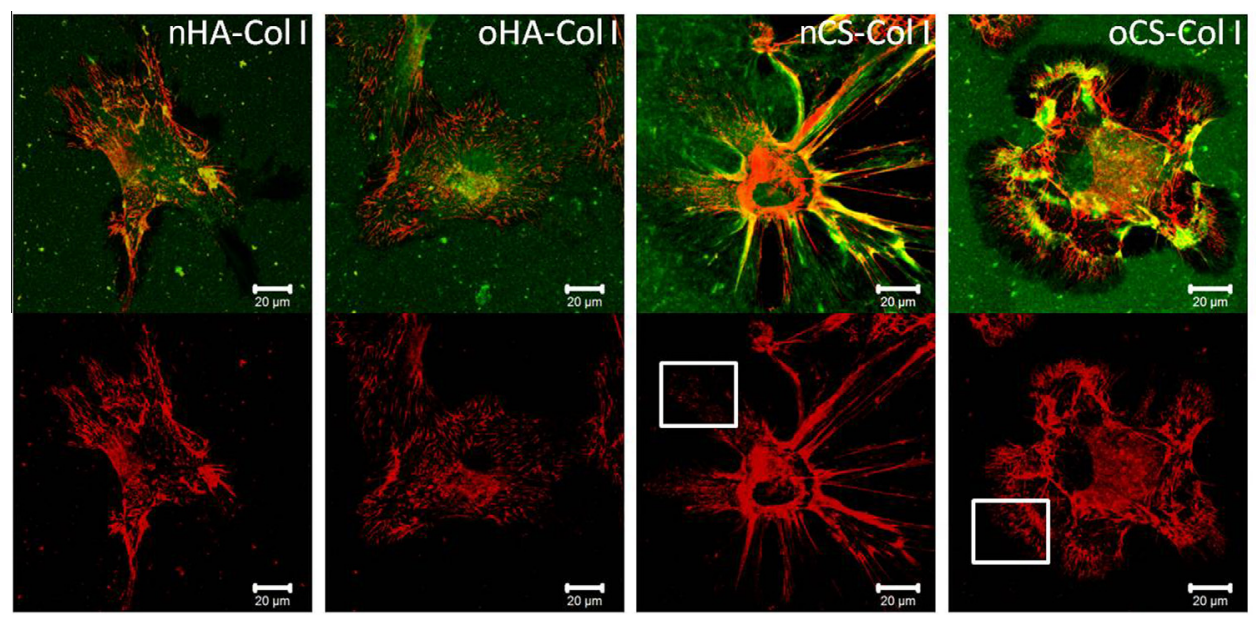

\section{Merge}

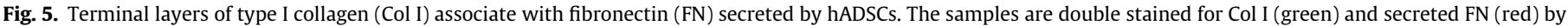

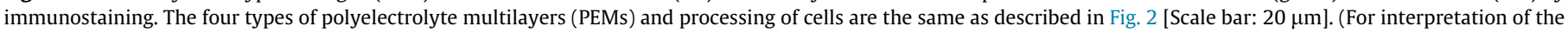
references to colour in this figure legend, the reader is referred to the web version of this article.)

activity by evaluating the release of labelled FITC-Col I (or its fragments) into the supernatant.

Fig. 6 shows the fluorescent signal intensity of released FITClabelled Col I measured under different conditions, including: (A) spontaneous release of FITC-Col I into the medium from samples with cells $(+)$ or without cells $(-)$, and (B) fluorescent signal after extraction of FITC-Col I with $\mathrm{NaOH}$ from the same samples, again in the presence $(+)$ or absence $(-)$ of cells, characterizing the 
A

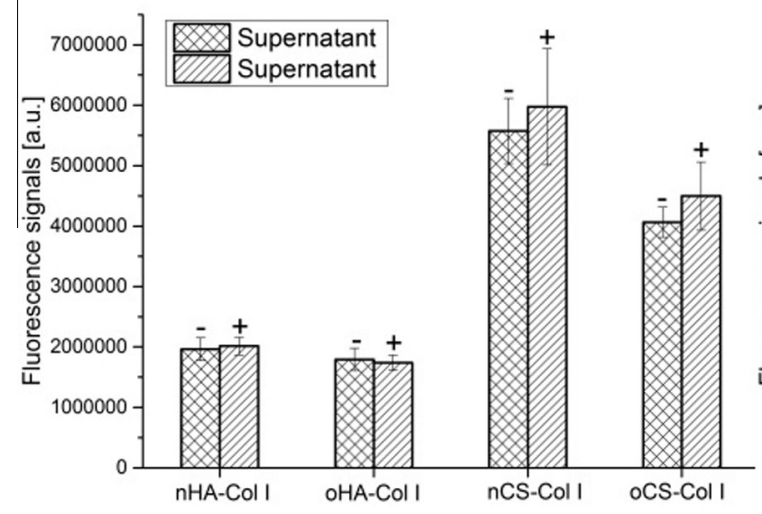

B

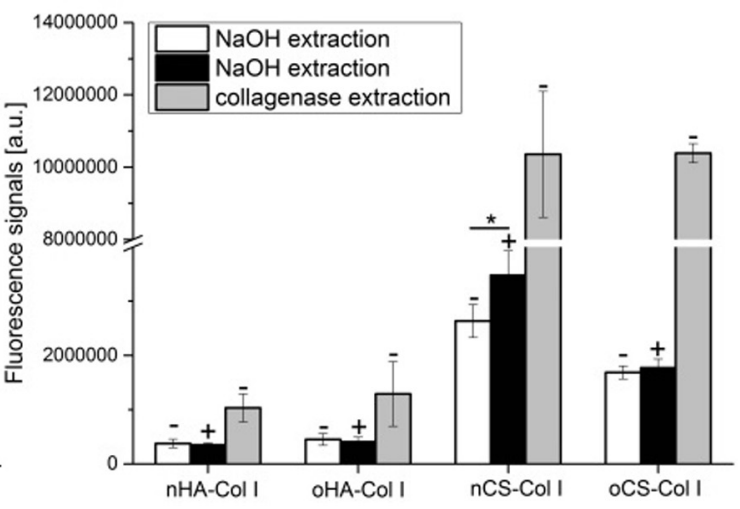

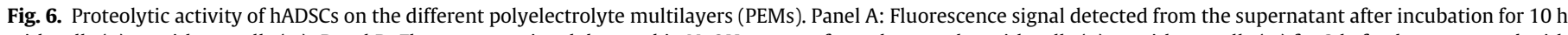

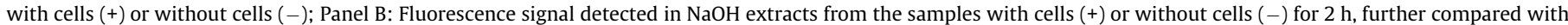

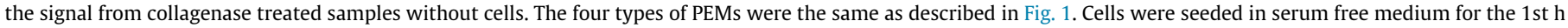
and then the medium was supplied with $10 \%$ serum for additional $10 \mathrm{~h}$ of incubation.

de-quenching of substratum bound FITC-Col I (see Methods section). The maximal de-quenching (e.g. complete proteolysis) caused by the exposure of a given sample to collagenase is also shown for comparison. Fig. 6 A shows that the release of FITC-Col I from all PEM systems after $10 \mathrm{~h}$ incubation did not differ significantly, either with or without cells, though a little tendency $(p>0.05)$ of increased release was detected in samples "+ cells" for CS-based PEMs. A significantly higher fluorescence signal was found for CS-based PEM systems, which correlates well with the higher Col I content measured by BCA assay. It was also observed that the spontaneous release from nCS-based PEMs was higher than from oCS-based one, which is another hint for the increased stability due intrinsic cross-linking by imine bond formation.

Fig. 6B shows the NaOH-extracted FITC-Col I fluorescent signal from the samples "with cells" $(+)$ versus "without cells" $(-)$ compared to the maximal signal from collagenase treated samples. No effect of the adhering cells was observed in HA-based PEM systems suggesting an absent or negligible proteolytic activity in comparison to the results obtained with collagenase treated PEMs (positive control). However, a significant increase of the signal " $(+)$ cells" versus $(-)$ cells $(\mathrm{p}<0.05)$ was found in nCS-containing PEMs, suggesting a valuable proteolytic activity of hADSCs against $\mathrm{Col} \mathrm{I}$ in this system. Again absent cell-mediated proteolysis was found in oCS-based PEMs, which seems to be again related to the enhanced stability of cross-linked PEMs.

\subsection{Cell proliferation}

The proliferation of hADSCs over a period of 1,2 and 3 days on the different PEMs was studied with QBlue assay, which determines the metabolic activity of cells (Fig. 7). In correspondence to the cell adhesion results, there was no significant difference in the metabolic activity of cells on different PEMs after one day of incubation. However, a significantly higher metabolic activity $(\mathrm{p}<0.05)$ was found on CS-based PEMs compared to HA-based ones at longer incubation times, though no significant difference was found between the PEMs of the same type of GAG (as shown in Fig. 7). It is well documented that integrin-mediated cell spreading and FA formation triggers cell proliferation and support the survival of anchorage dependent cells due to activation of MAP kinase pathway and up-regulation of Bcl-2 family of proteins [55]. Integrin-mediated cell spreading and FA formation was more pronounced on CS-based PEMs, obviously providing better conditions for cell growth than PEMs based on HA (as shown in Fig. 2

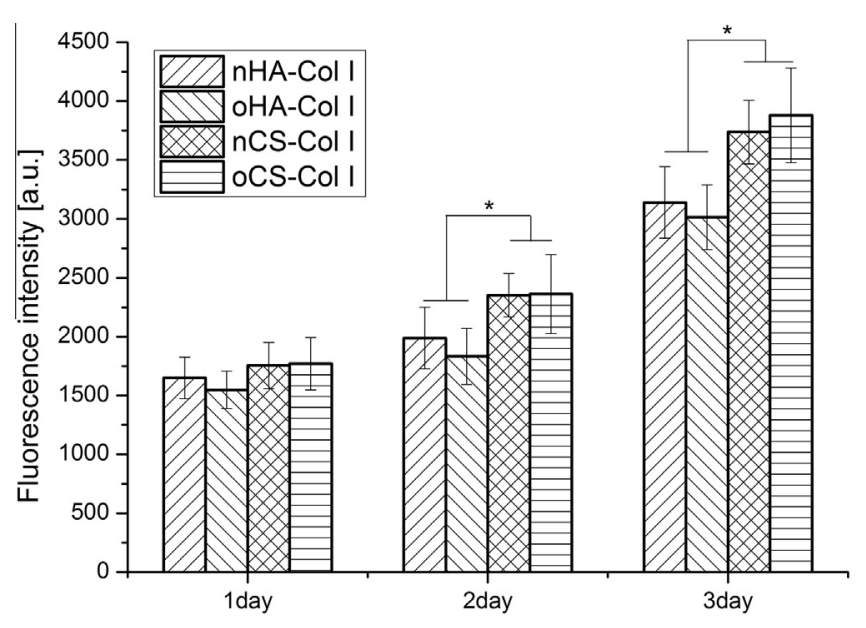

Fig. 7. Proliferation of hADSCs plated on different surfaces assessed by QBlue assay related to the metabolic active cells during the culture time (1,2 and 3 days). Results are means \pm SD of three independent experiments. The four types of polyelectrolyte multilayers (PEMs) are the same as described in Fig. 2.

and Fig. 3). Furthermore, the ability of cells to develop a structured ECM at the materials interface was also supported particularly in nCS but also on oCS-based PEMs, which seems to promote further the activity of cells as shown in previous studies for other type of biomaterials systems $[8,53]$.

\subsection{Osteogenic differentiation of hADSCS}

The activity of ALP is an important prerequisite for mineral deposition by osteoblasts, since the enzyme catalyzes the release of phosphate from phosphate donors for subsequent precipitation of calcium ions and formation of hydroxyapatite [56]. To evaluate the function of hADSCs on the various PEMs, ALP activity was normalized to protein content and monitored at day 5,11 and 15 postdifferentiation (Fig. 8). There was no detectable difference of ALP activity of hADSCs cultured in BM on the different PEMs. However, ALP activity of hADSCs increased largely after addition of OM between days 5 and 15 on all the test samples (Fig. 8). In contrast to this, only a slight increase of ALP activity within 15 days was detected, when cells were cultured in BM. Notably, pronounced differences were found when comparing the ALP activity of hADSCs cultured in OM on the four types of PEMs. As shown in 


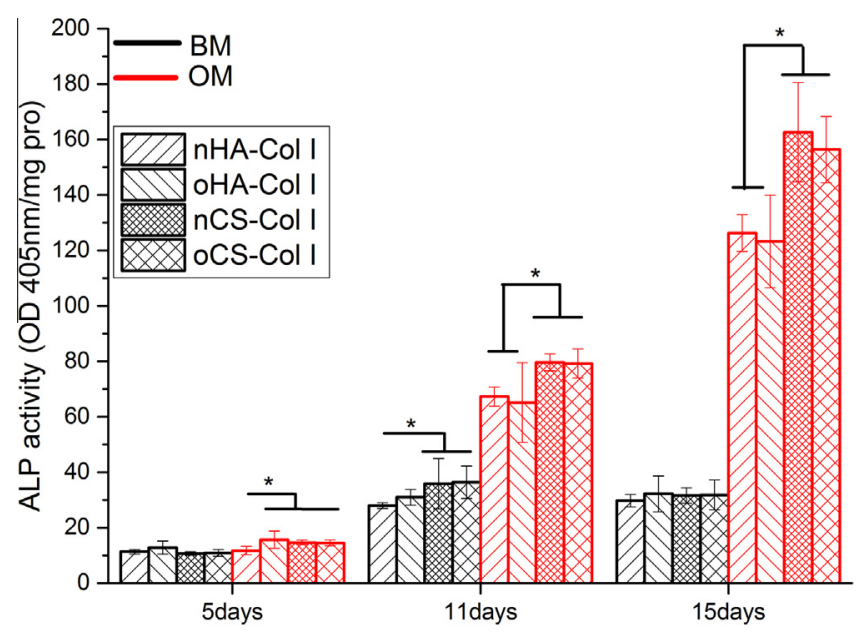

Fig. 8. Activity of alkaline phosphates (ALP) normalized to protein content of hADSCs plated on the different polyelectrolyte multilayers (PEMs) after 3 weeks induction with basal medium (BM) and osteogenic differentiation medium (OM), respectively. ALP activity was determined after lysis of cells and use of pnitrophenylphosphate ( $\mathrm{pNPP}$ ) as substrate, normalized to the protein content of the lysates. The four types of PEMs were the same as described in Fig. 2.

Fig. 8, both CS-based PEMs showed a significantly higher ALP activity compared to HA-containing PEMs within the culture time, suggesting that CS-based PEMs promote the osteogenic differentiation of hADSCs.

The deposition of hydroxyapatite is one feature of mature osteoblasts. hADSCs cultured on the different PEMs in BM or OM were stained at day 21 post-differentiation with Alizarin Red S to visualize calcium phosphate deposition (see Fig. 9). No significant staining was observed when hADSCs were cultured in BM (upper panel of Fig. 9) indicating the need of osteogenic inductors for differentiation of these stem cells. However, a staining was observed on all PEMs when hADSCs were cultured in OM (lower panel of Fig. 9), although the staining was more intense on CS-based compared to HA-based PEMs. Specifically, oCS-based PEMs induced most extensive calcium deposition, indicating that the intrinsic cross-linking of PEMs may enhance the osteogenic differentiation of hADSCs.

Immunofluorescence staining shown in Fig. $10 \mathrm{~A}$ was also used to detect de novo expression of the bone-specific ECM protein Col I (green staining) synthesized by hADSCs (blue nuclear staining) cultured in either BM or OM after 21 days induction. The upper panel of Fig. 10A shows micrographs from hADSCs cultured in BM, where no visible staining of Col I was observed. This finding demonstrates not only that osteogenic inductors are required for hADSCs, but also that the Col I initially deposited during PEMs formation did not contribute to staining after this time. By contrast, intense staining with visualization of a fibrillar structure of newly synthesized Col I became visible particularly when cells were cultured on CSbased multilayers in OM (lower panel in Fig. 10A). It was also evident here in a qualitative manner that oCS-based PEMs promoted most Col I synthesis, as a more extended network of Col I was detected here. To further study the effect of molecular composition of PEMs on the differentiation potential of hADSCs, the osteogenic markers Runx2 and Col I were measured by qRT-PCR at day 14 post-differentiation. As shown in Fig. 10B, CS-based PEMs especially the oCS-PEMs caused a significant up-regulation of Runx2 expression in comparison to HA-containing PEMs, when hADSCs were cultured in OM. Runx2 is a zinc finger transcription factor, which is essential for osteoblast differentiation, acting on the downstream signaling and promoting the expression of important osteoblast proteins, such as Col I. Notably, a pronounced increase of Col I expression was observed in hADSCs cultured on oCSbased PEMs, however, no detectable differences were found among the other three groups (see Fig. 10C).

ALP activity test, histochemical staining, immunofluorescence staining, and qRT-PCR results demonstrate that CS-containing PEMs, particularly oCS-based one greatly enhance hADSCs osteogenic differentiation compared to cells on HA-containing PEMs in the presence of osteogenic media supplements. The reason for superior activity of CS-based PEMs is probably the larger quantity and fibrillar structure of Col I, which is also a major component of bone tissue. This was also evident here by enhanced the cell matrix interactions, such as FA formation and integrin clustering. It is well in documented that osteogenesis is mediated by integrins, with $\beta 1$ and $\beta 3$ integrins each having been found to be important in regulating the osteogenic differentiation of hMSCs [57]. Interaction of hMSCs with Col I occurs via $\beta 1$ integrins and has been shown to be critical for their osteogenic differentiation when cultured on Col I matrix including phosphorylation of focal adhesion kinase (FAK), downstream signaling by MAP kinase related to expression of osteogenic transcription factors [58,59]. On the other hand, many studies demonstrated the beneficial of CS on bone formation $[60,61]$. Murphy and the co-workers found that the type of GAG used, can control the lineage specification of MSCs, whereby HA enhanced chondrogenic differentiation and CS promoted osteogenic MSC differentiation [61]. Furthermore, the CS-based PEMs

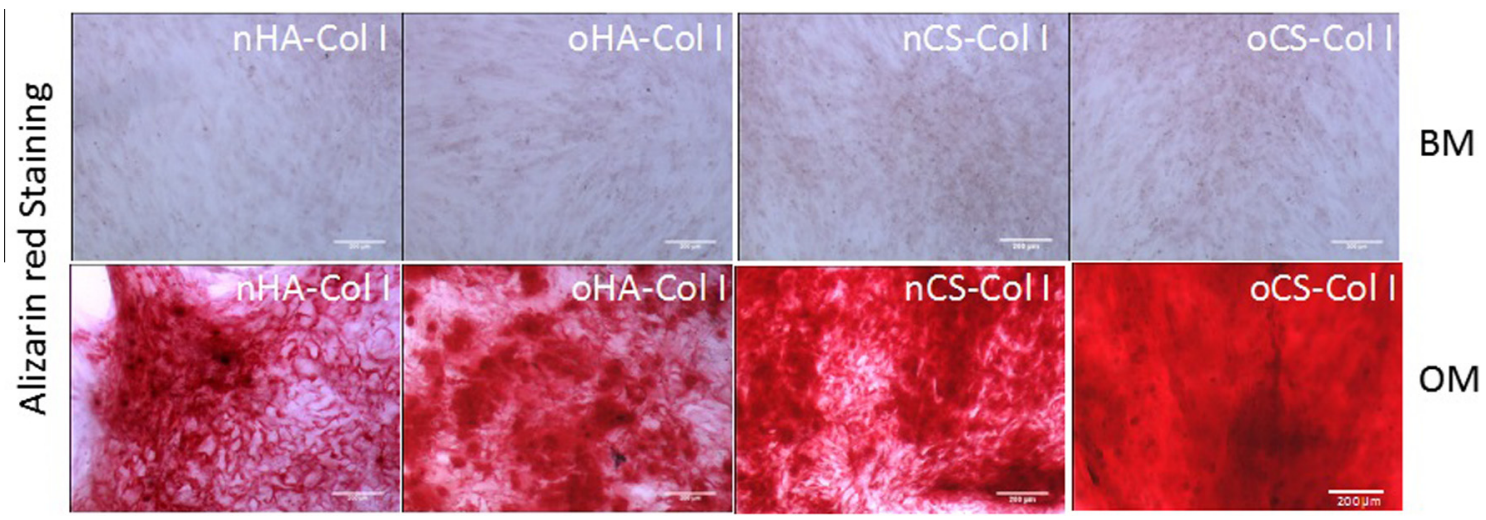

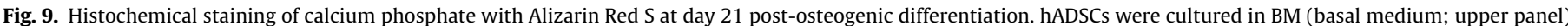

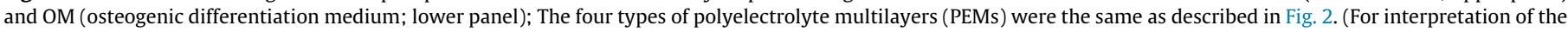
references to colour in this figure legend, the reader is referred to the web version of this article.) 

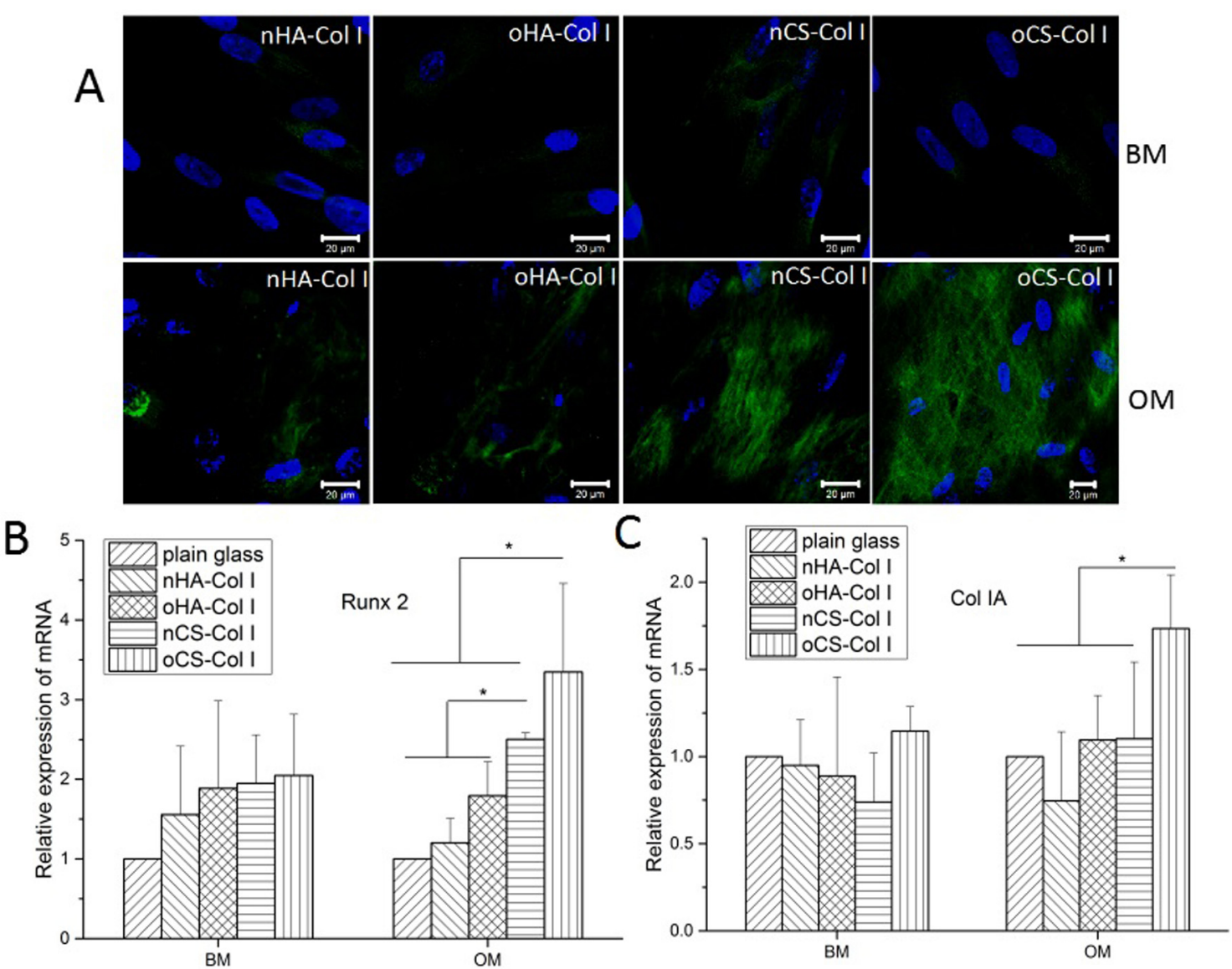

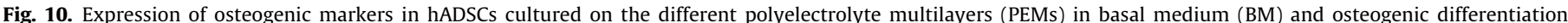

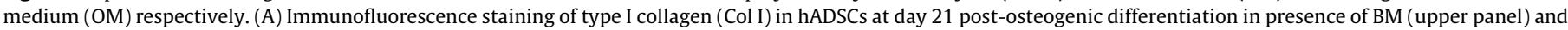

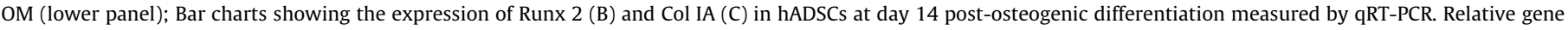
expression is presented as normalized to gene expression by hADSCs cultured on plain glass. The four types of PEMs were the same as described in Fig. 2.

permitted a significant remodeling of the terminal Col I layer, which allowed cells to develop a provisional ECM, including an extensive secretion of FN, which may further support ligation of integrins and signal transduction processes eventually supporting the osteogenic differentiation of hADSCs, as well. The most pronounced osteogenesis observed on the oCS-based PEMs might be caused by the improved stability of the biogenic components of these multilayers, which have then a long term effect on hADSCs differentiation.

\section{Conclusion}

This work demonstrates the impact of the microenvironment on stem cell fate during in vitro culture of hADSCs on PEMs made of Col I and either HA or CS by layer-by-layer (LbL) technique. By varying the type of GAG, we showed that the initial cellular behavior, and hence the fate of hADSCs regarding osteogenic differentiation was significantly affected. The CS-based PEMs that were constituted of more and fibrillary organized Col I supported hADSCs osteogenesis most, which was related to an increased cell spreading, FA formation, pronounced integrin clustering and welldefined actin cytoskeleton organization at early stages of culture, followed by more growth and osteogenic differentiation of hADSCs. It was also found that intrinsic cross-linking of oCS-based PEMs amplified the osteogenic differentiation, which might be related to the higher stiffness of this multilayer system [22]. This study also shows for the first time an active remodeling of biogenic PEMs by adhering cells, based on both mechanical reorganization and pericellular proteolytic degradation, which obviously allowed further beneficial changes of the microenvironment of stem cells. Indeed, both mechanical remodeling and proteolysis were mainly restricted to terminal Col I layers, while leaving layers underneath unaffected. Hence, such systems might be also used for uploading bioactive factors to achieve a further multi-functionality of PEMs. These findings provide new insights to our understanding not only on the importance of controlling matrix composition as a strategy to manipulate stem cell fates, but also in the dynamic nature of PEMs in contact with cells, which may open new avenues for their application in tissue engineering and regenerative medicine. In addition, the use of intrinsic cross-linking systems based on oxidized GAGs and polyamines like collagen, but also chitosan can be also used to encapsulate cells and cytokines for making in situ forming 3D systems as shown in a recent publication [62], which represents a further promising application in engineering of bone, cartilage and other tissues.

\section{Diclosure}

No conflicts of interests are declared.

\section{Acknowledgments}

The work was funded partly by the European Commission, (FP7-PEOPLE-2012-IAPP) under grant agreement no. 324386 (FIBROGELNET), the EuroNanoMed project STRUCTGEL and the Chinese Scholarship Council program funded by Chinese government. 
Parts of the studies were supported by CIBER-BBN, the project MAT2012-38359-C03-03 HEALINSYNERGY funded by Spanish Ministry of Science and Innovation, the Medical Scientific Research Foundation of Guangdong Province, China (A2015341), and the PhD Start-up Fund of Affiliated Hospital of Guangdong Medical University, China (BJ201501). We are thankful to Prof. Changren Zhou and Prof. Lihua Li from Department of Materials Science and Engineering at Jinan University for providing type I collagen.

\section{Appendix A. Supplementary data}

Supplementary data associated with this article can be found, in the online version, at http://dx.doi.org/10.1016/j.actbio.2016.05. 023.

\section{References}

[1] S. Özbek, P.G. Balasubramanian, R. Chiquet-Ehrismann, R.P. Tucker, J.C. Adams, The evolution of extracellular matrix, Mol. Biol. Cell 21 (24) (2010) 4300-4305.

[2] F.M. Watt, W.T.S. Huck, Role of the extracellular matrix in regulating stem cell fate, Nat. Rev. Mol. Cell Biol. 14 (8) (2013) 467-473.

[3] K.A. Moore, I.R. Lemischka, Stem cells and their niches, Science 311 (5769) (2006) 1880-1885.

[4] H. Kresse, E. Schönherr, Proteoglycans of the extracellular matrix and growth control, J. Cell. Physiol. 189 (3) (2001) 266-274.

[5] Z.-M. Liu, Q. Gu, Z.-K. Xu, T. Groth, Synergistic effect of polyelectrolyte multilayers and osteogenic growth medium on differentiation of human mesenchymal stem cells, Macromol. Biosci. 10 (9) (2010) 1043-1054.

[6] J.E. Phillips, T.A. Petrie, F.P. Creighton, A.J. García, Human mesenchymal stem cell differentiation on self-assembled monolayers presenting different surface chemistries, Acta Biomater. 6 (1) (2010) 12-20.

[7] R.O. Hynes, Extracellular matrix: not just pretty fibrils, Science 326 (5957) (2009) 1216-1219.

[8] W.P. Daley, S.B. Peters, M. Larsen, Extracellular matrix dynamics in development and regenerative medicine, J. Cell Sci. 121 (3) (2008) 255-264.

[9] L. Maneva-Radicheva, U. Ebert, N. Dimoudis, G. Altankov, Fibroblast remodeling of adsorbed collagen type IV is altered in contact with cancer cells, Histol. Histopathol. 23 (7) (2008) 833-842.

[10] E. Tamariz, F. Grinnell, Modulation of fibroblast morphology and adhesion during collagen matrix remodeling, Mol. Biol. Cell 13 (11) (2002) 3915-3929.

[11] N.M. Coelho, M. Salmeron-Sanchez, G. Altankov, Fibroblasts remodeling of type IV collagen at a biomaterials interface, Biomater. Sci. 1 (5) (2013) 494502.

[12] M.M. Giraud Guille, G. Mosser, Fau C. Helary, D. Eglin, Bone matrix like assemblies of collagen: from liquid crystals to gels and biomimetic materials, Micron 36 (7-8) (2005) 602-608.

[13] N. Matsumoto, S. Horibe, N. Nakamura, T. Senda, K. Shino, T. Ochi, Effect of alignment of the transplanted graft extracellular matrix on cellular repopulation and newly synthesized collagen, Arch. Orthop. Trauma Surg. 117 (4-5) (1998) 215-221.

[14] D. He, X. Xiao, F. Liu, R. Liu, Chondroitin sulfate template-mediated biomimetic synthesis of nano-flake hydroxyapatite, Appl. Surf. Sci. 255 (2) (2008) 361 364.

[15] Y. Liu, X. Wang, D.S. Kaufman, W. Shen, A synthetic substrate to support early mesodermal differentiation of human embryonic stem cells, Biomaterials 32 (32) (2011) 8058-8066

[16] X.-D. Chen, Extracellular matrix provides an optimal niche for the maintenance and propagation of mesenchymal stem cells, Birth Defect. Res. C Embryo Today. 90 (1) (2010) 45-54.

[17] P.A. Zuk, M. Zhu, H. Mizuno, J. Huang, J.W. Futrell, A.J. Katz, P. Benhaim, H.P. Lorenz, M.H. Hedrick, Multilineage cells from human adipose tissue: implications for cell-based therapies, Tissue Eng. 7 (2) (2001) 211-228.

[18] J.M. Hong, B.J. Kim, J.-H. Shim, K.S. Kang, K.-J. Kim, J.W. Rhie, H.J. Cha, D.W. Cho, Enhancement of bone regeneration through facile surface functionalization of solid freeform fabrication-based three-dimensional scaffolds using mussel adhesive proteins, Acta Biomater. 8 (7) (2012) 2578-2586.

[19] H. Shin, S. Jo, A.G. Mikos, Biomimetic materials for tissue engineering, Biomaterials 24 (24) (2003) 4353-4364.

[20] E. Rodriguez-Velazquez, M. Alatorre-Meda, J.F. Mano, Polysaccharide-based nanobiomaterials as controlled release systems for tissue engineering applications, Curr. Pharm. Des. 21 (33) (2015) 4837-4850.

[21] M.B. Rahmany, M. Van Dyke, Biomimetic approaches to modulate cellular adhesion in biomaterials: a review, Acta Biomater. 9 (3) (2013) 5431-5437.

[22] M. Zhao, L. Li, C. Zhou, F. Heyroth, B. Fuhrmann, K. Maeder, T. Groth, Improved stability and cell response by intrinsic cross-linking of multilayers from collagen i and oxidized glycosaminoglycans, Biomacromolecules 15 (11) (2014) 4272-4280.

[23] S.M. Oliveira, V.E. Santo, M.E. Gomes, R.L. Reis, J.F. Mano, Layer-by-layer assembled cell instructive nanocoatings containing platelet lysate, Biomaterials 48 (2015) 56-65.
[24] R.F. Mhanna, J. Vörös, M. Zenobi-Wong, Layer-by-layer films made from extracellular matrix macromolecules on silicone substrates, Biomacromolecules 12 (3) (2011) 609-616.

[25] C. Chaubaroux, E. Vrana, C. Debry, P. Schaaf, B. Senger, J.-C. Voegel, Y. Haikel, C. Ringwald, J. Hemmerlé, P. Lavalle, F. Boulmedais, Collagen-based fibrillar multilayer films cross-linked by a natural agent, Biomacromolecules 13 (7) (2012) 2128-2135.

[26] W.H. Ko, J.T. Suminto, G.J. Yeh, Micromachining and Micropackaging of Transducers, Elsevier, Amsterdam, The Netherlands, 1985, pp. 41-61.

[27] G. Altankov, F. Grinnell, T. Groth, Studies on the biocompatibility of materials: fibroblast reorganization of substratum-bound fibronectin on surfaces varying in wettability, J. Biomed. Mater. Res. 30 (3) (1996) 385-391.

[28] R.A. McCloy, S. Rogers, C.E. Caldon, T. Lorca, A. Castro, A. Burgess, Partial inhibition of Cdk1 in G2 phase overrides the SAC and decouples mitotic events, Cell Cycle 13 (9) (2014) 1400-1412.

[29] M. Ochsner, M. Textor, V. Vogel, M.L. Smith, Dimensionality controls cytoskeleton assembly and metabolism of fibroblast cells in response to rigidity and shape, PLoS ONE 5 (3) (2010) e9445.

[30] F. Bathawab, M. Bennett, M. Cantini, J. Reboud, M.J. Dalby, M. SalmeronSanchez, Lateral chain length in polyalkyl acrylates determines the mobility of fibronectin at the cell/material interface, Langmuir 32 (3) (2016) 800-809.

[31] B.S. McSwain, R.L. Irvine, M. Hausner, P.A. Wilderer, Composition and distribution of extracellular polymeric substances in aerobic flocs and granular sludge, Appl. Environ. Microbiol. 71 (2) (2005) 1051-1057.

[32] P. Chomczynski, N. Sacchi, Single-step method of RNA isolation by acid guanidinium thiocyanate-phenol-chloroform extraction, Nat. Protoc. 1 (2) (2006) 581-585.

[33] K.J. Livak, T.D. Schmittgen, Analysis of relative gene expression data using realtime quantitative PCR and the 2(-Delta Delta C(T)) method, Methods 25 (4) (2001) 402-408.

[34] V. Gribova, R. Auzely-Velty, C. Picart, Polyelectrolyte multilayer assemblies on materials surfaces: from cell adhesion to tissue engineering, Chem. Mater. 24 (5) (2012) 854-869.

[35] N. Aggarwal, N. Altgärde, S. Svedhem, K. Zhang, S. Fischer, T. Groth, Effect of molecular composition of heparin and cellulose sulfate on multilayer formation and cell response, Langmuir 29 (45) (2013) 13853-13864.

[36] J.F.L. Duval, D. Küttner, C. Werner, R. Zimmermann, Electrohydrodynamics of soft polyelectrolyte multilayers: point of zero-streaming current, Langmuir 27 (17) (2011) 10739-10752.

[37] B.C. Wood, The Formation of Fibrils from Collagen Solutions 3. Effect of chondroitin sulfate and some other naturally occurring polyanions on the rate of formation, Biochem. J. 75 (3) (1960) 605-612.

[38] A.J. Kvist, A.E. Johnson, M. Mörgelin, E. Gustafsson, E. Bengtsson, K. Lindblom, A. Aszódi, R. Fässler, T. Sasaki, R. Timpl, A. Aspberg, Chondroitin sulfate perlecan enhances collagen fibril formation. Implications for perlecan chondrodysplasias, J. Biol. Chem. 281 (44) (2006) 33127-33139.

[39] F. Jiang, H. Hörber, J. Howard, D.J. Müller, Assembly of collagen into microribbons: effects of $\mathrm{pH}$ and electrolytes, J. Struct. Biol. 148 (3) (2004) 268-278.

[40] M. Raspanti, M. Viola, M. Sonaggere, M.E. Tira, R. Tenni, Collagen fibril structure is affected by collagen concentration and decorin, Biomacromolecules 8 (7) (2007) 2087-2091.

[41] F. Gobeaux, G. Mosser, A. Anglo, P. Panine, P. Davidson, M.M. Giraud-Guille, E. Belamie, Fibrillogenesis in dense collagen solutions: a physicochemical study, J. Mol. Biol. 376 (5) (2008) 1509-1522.

[42] R.M. Ezzell, W.H. Goldmann, N. Wang, N. Parasharama, D.E. Ingber, Vinculin promotes cell spreading by mechanically coupling integrins to the cytoskeleton, Exp. Cell Res. 231 (1) (1997) 14-26.

[43] R.O. Hynes, Integrins: bidirectional, allosteric signaling machines, Cell 110 (6) (2002) 673-687.

[44] J. Khoshnoodi, V. Pedchenko, B.G. Hudson, Mammalian collagen IV, Microsc. Res. Tech. 71 (5) (2008) 357-370.

[45] N.M. Coelho, C. Gonzalez-Garcia, J.A. Planell, M. Salmeron-Sanchez, G. Altankov, Different assembly of type IV collagen on hydrophilic and hydrophobic substrata alters endothelial cells interaction, Eur. Cell Mater. 19 (2010) 262-272.

[46] K.B. Whitson, A.M. Lukan, R.L. Marlowe, S.A. Lee, L. Anthony, A. Rupprecht, Binding of the water of primary hydration to the sodium and cesium salts of deoxyribonucleic acid and potassium hyaluronate, Phys. Rev. E 58 (2) (1998) 2370-2377.

[47] L. Richert, A.J. Engler, D.E. Discher, C. Picart, Elasticity of native and crosslinked polyelectrolyte multilayer films, Biomacromolecules 5 (5)(2004) 19081916.

[48] C. Picart, Polyelectrolyte multilayer films: from physico-chemical properties to the control of cellular processes, Curr. Med. Chem. 15 (7) (2008) 685-697.

[49] M.P. Lutolf, J.A. Hubbell, Synthetic biomaterials as instructive extracellular microenvironments for morphogenesis in tissue engineering, Nat. Biotechnol. 23 (1) (2005) 47-55.

[50] Y. Mao, J.E. Schwarzbauer, Fibronectin fibrillogenesis, a cell-mediated matrix assembly process, Matrix Biol. 24 (6) (2005) 389-399.

[51] T. Velling, J. Risteli, K. Wennerberg, D.F. Mosher, S. Johansson, Polymerization of Type I and III collagens is dependent on fibronectin and enhanced by integrins $\alpha 11 \beta 1$ and $\alpha 2 \beta 1$, J. Biol. Chem. 277 (40) (2002) 37377-37381.

[52] K.E. Kadler, A. Hill, E.G. Canty-Laird, Collagen fibrillogenesis: fibronectin, integrins, and minor collagens as organizers and nucleators, Curr. Opin. Cell Biol. 20 (5) (2008) 495-501. 
[53] M. Larsen, V.V. Artym, J.A. Green, K.M. Yamada, The matrix reorganized: extracellular matrix remodeling and integrin signaling, Curr. Opin. Cell Biol. 18 (5) (2006) 463-471.

[54] F. Grinnell, Focal adhesion sites and the removal of substratum-bound fibronectin, J. Cell Biol. 103 (6) (1986) 2697-2706.

[55] R.O. Hynes, Integrins: versatility, modulation, and signaling in cell adhesion, Cell 69 (1) (1992) 11-25.

[56] H. Orimo, The mechanism of mineralization and the role of alkaline phosphatase in health and disease, J. Nippon Med. Sch. 77 (1) (2010) 4-12.

[57] A. Shekaran, A.J. García, Extracellular matrix-mimetic adhesive biomaterials for bone repair, J. Biomed. Mater. Res. A. 96A (1) (2011) 261-272.

[58] A.W. Lund, J.P. Stegemann, G.E. Plopper, Inhibition of ERK promotes collagen gel compaction and fibrillogenesis to amplify the osteogenesis of human mesenchymal stem cells in three-dimensional collagen I culture, Stem Cell Dev. 18 (2) (2009) 331-341.
[59] R.M. Salasznyk, R.F. Klees, M.K. Hughlock, G.E. Plopper, ERK signaling pathways regulate the osteogenic differentiation of human mesenchymal stem cells on collagen I and vitronectin, Cell Commun. Adhes. 11 (5-6) (2004) $137-153$.

[60] S. Rammelt, T. Illert, S. Bierbaum, D. Scharnweber, H. Zwipp, W. Schneiders, Coating of titanium implants with collagen, RGD peptide and chondroitin sulfate, Biomaterials 27 (32) (2006) 5561-5571.

[61] C.M. Murphy, A. Matsiko, M.G. Haugh, J.P. Gleeson, F.J. O’Brien, Mesenchymal stem cell fate is regulated by the composition and mechanical properties of collagen- glycosaminoglycan scaffolds, J. Mech. Behav. Biomed. Mater. 11 (2012) 53-62.

[62] C. Millan, E. Cavalli, T. Groth, K. Maniura-Weber, M. Zenobi-Wong, Engineered microtissues formed by schiff base crosslinking restore the chondrogenic potential of aged mesenchymal stem cells, Adv. Funct. Mater. 4 (9) (2015) 1348-1358. 